\title{
Avaliação do conhecimento de estudantes da área da saúde sobre a Escala de Coma de Glasgow em uma Universidade de Minas Gerais
}

\author{
Health students' knowledge assessment on the Glasgow Coma Scale at the University of Minas \\ Gerais \\ Evaluación del conocimiento de los estudiantes de salud sobre la Escada de Coma de Glasgow en una \\ Universidad de Minas Gerais
}

\section{Resumo}

Introdução: os estudantes da área da saúde precisam ter pleno conhecimento dos métodos de avaliação de consciência para excelência do exercício profissional e atendimento ao paciente, no entanto a literatura relata deficiências dos profissionais de saúde na utilização da Escala de Coma de Glasgow (ECG). Objetivo: o objetivo do presente estudo foi analisar e avaliar o conhecimento de estudantes da área da saúde sobre a função e aplicabilidade da Escala de Coma de Glasgow (ECG). Métodos: os dados foram gerados pela aplicação de questionário aos acadêmicos do último ano dos cursos de Medicina, Enfermagem, Odontologia e Farmácia da Universidade Federal de Alfenas. Resultados e Discussão: os resultados revelam que os futuros médicos e enfermeiros apresentam maior conhecimento dos parâmetros e aplicação da ECG quando comparado aos demais estudantes avaliados, isso é um empecilho para excelência do cuidado em saúde, visto que as equipes multiprofissionais são de extrema importância para o cuidado, e seus membros necessitam de um conhecimento ampliado sobre ECG para fornecer ao paciente um monitoramento seriado e evitar problemas de comunicação entre a equipe. Conclusão: ocorre um déficit de aprendizado sobre a ECG entre os estudantes da área da saúde e para supri-lo as universidades devem se comprometer com a oferta de cursos teóricos e práticos sobre o uso da ECG.

Palavras-chave: Escala de Coma de Glasgow; Educação em saúde; Equipe multiprofissional.

\begin{abstract}
Introduction: health care students need to have full knowledge of awareness assessment methods for excellence in professional practice and patient care, however, the literature reports health professionals' shortcomings in the use of the Glasgow Coma Scale (ECG). Objective: The aim of the present study was to analyze and assess the knowledge of healthcare students about the function and applicability of the Glasgow Coma Scale (ECG). Methods: data were generated by applying a questionnaire to senior students of Medicine, Nursing, Dentistry and Pharmacy courses at the Federal University of Alfenas. Results and Discussion: the results reveal that future doctors and nurses have greater knowledge of the parameters and application of ECG when compared to other evaluated students, this is an obstacle to excellence in health care, since multidisciplinary teams are extremely important for care, and its members need an expanded knowledge of ECG to provide the patient with serial monitoring and avoid communication problems between the team. Conclusion: there is a deficit in learning about ECG among students in the health field and, in order to supply it, universities must commit to offering theoretical and practical courses on the use of ECG.
\end{abstract}

Keywords: Glasgow Coma Scale; Health education; Multidisciplinary team.

\section{Resumen}

Introducción: los estudiantes de salud deben tener un conocimiento completo de los métodos de evaluación de la conciencia para la excelencia en la práctica profesional y la atención al paciente, sin embargo, la literatura reporta las deficiencias de los profesionales de la salud en el uso de la Escala de coma de Glasgow (ECG). Objetivo: El objetivo 
del presente estudio fue analizar y evaluar el conocimiento de los estudiantes de la salud sobre la función y aplicabilidad de la Escala de Coma de Glasgow (ECG). Métodos: los datos se generaron mediante la aplicación de un cuestionario a estudiantes de último año de los cursos de Medicina, Enfermería, Odontología y Farmacia de la Universidad Federal de Alfenas. Resultados y Discusión: los resultados muestran que los futuros médicos y enfermeras tienen un mayor conocimiento de los parámetros y aplicación del ECG en comparación con otros estudiantes evaluados, esto es un obstáculo para la excelencia en la atención de la salud, ya que los equipos multidisciplinarios son de suma importancia para la atención, y su Los miembros necesitan un conocimiento más amplio de ECG para proporcionar al paciente un seguimiento en serie y evitar problemas de comunicación entre el equipo. Conclusión: existe un déficit en el aprendizaje del ECG entre los estudiantes del campo de la salud y, para suplirlo, las universidades deben comprometerse a ofrecer cursos teóricos y prácticos sobre el uso del ECG.

Palabras clave: Escala de Coma de Glasgow; Educación para la salud; Equipo multidisciplinario.

\section{Introdução}

A escala de coma de Glasgow (ECG), desenvolvida na Universidade de Glasgow em 1974 na Escócia por Taesdale e Jennett (Jennett \& Teasdale, 1977), é um instrumento neurológico utilizado mundialmente para avaliar o nível de consciência (Fisher \& Mathieson, 2001; Jain \& Iverson, 2021).

Barata e eficaz, foi a primeira escala padronizada e de importância para avaliação da consciência em pacientes traumatizados (Ross et al., 1998; Ingram, 1994; Mcnarry \& Goldhill, 2004; Jain \& Iverson, 2021). Avaliando os aspectos da consciência de excitação e cognição indiretamente por meio de observação da resposta perante os diferentes estímulos (Stewart, 1996; Shah, 1999; Hickey, 1997), onde a excitação mede a consciência do ambiente e a cognição demonstra a compreensão do meio pela habilidade de realizar tarefas (Edwards, 2001).

A escala é baseada nos comportamentos de melhor resposta motora, verbal e ocular advinda do paciente (Emeleju, Nkwerem \& Ekweogwu, 2014; Shoqirat, 2006; Jain \& Iverson, 2021) (Tabela 1), e baseado nisto são atribuídos pontos as diferentes respostas, sendo o nível de consciência avaliado de 3 a 15 (Bryan, 2002; Jain \& Iverson, 2021), no qual o score 3 indica um coma profundo e o score 15 indica ausência de dano neurológico (Mattar, Liaw \& Chan, 2013).

A pontuação é utilizada para triagem do trauma e norteia a conduta médica (Jain \& Iverson, 2021), além de servir para monitoramento de pacientes com nível de consciência afetado por afecções variadas, como metabólicas, neoplásicas, degenerativas (Emeleju, Nkwerem \& Ekweogwu, 2014; Baker, 2008; Fisher \& Mathieson, 2001; Ellis \& Cavanagh, 1992). Em 2018, após estudos de Teasdale, Brennan e Murray, foi adicionada a reatividade pupilar à avaliação, com o objetivo de inferir o prognóstico no traumatismo cranioencefálico (Brennan, Murray \& Teasdele, 2018). A avaliação da reatividade pupilar ocorre de forma que, a quantidade de pupilas não-fotorreagentes é subtraída da pontuação da ECG (ATLS, 2018). 
Tabela 1. Escala de Coma de Glasgow: Variáveis e Pontuação.

\begin{tabular}{c|cc}
\hline Variável & Resposta & Pontuação \\
\hline \multirow{3}{*}{ Melhor resposta ocular } & Espontânea & 4 \\
& À voz & 3 \\
& À dor & 2 \\
& Nenhuma & 1 \\
\hline \multirow{3}{*}{ Melhor Resposta Verbal } & Orientada & 5 \\
& Confusa & 4 \\
& Palavras inapropriadas & 3 \\
& Palavras incomprieensivas & 2 \\
& Nenhuma & 1 \\
\hline \multirow{3}{*}{ Melhor Resposta Motora } & Obedece a comandos & 5 \\
& Localiza a dor & 4 \\
& Movimento de retirada & 3 \\
& Flexão anormal (decorticação) & 2 \\
& Extensão anormal (descerebração) & 1 \\
\hline Inexistente & Nenhuma & 0 \\
\hline \multirow{2}{*}{-2} & Reatividade Pupilar & Bilateral \\
\hline
\end{tabular}

Fonte: adaptado de Jennett e Teasdale (1977), e ATLS (2018).

A utilização da ECG requer conhecimento prévio e habilidades (Jain \& Iverson, 2021). A falta de conhecimento sobre sua interpretação implica em imprecisão da avaliação do estado neurológico, podendo gerar ruídos na comunicação da equipe de saúde e, consequentemente, piora na qualidade do serviço prestado ao doente (Shoqirat, 2006), demonstrando, assim, a necessidade de conhecimento pleno dos profissionais e estudantes da área da saúde.

O objetivo deste estudo foi verificar o nível de conhecimento da escala de coma de Glasgow entre os estudantes do último ano de Medicina, Enfermagem, Odontologia e Farmácia da Universidade Federal de Alfenas.

\section{Metodologia}

Os participantes foram recrutados em setembro de 2019, após anuência dos Diretores das respectivas unidades e aprovação do Conselho de Ética em Pesquisa (CEP) da UNIFAL- MG. O critério de inclusão foi à seleção feita de forma aleatória entre acadêmicos do último ano dos cursos de Odontologia, Medicina, Farmácia e Enfermagem, totalizando 100 estudantes. Cada participante leu e assinou o Termo de Consentimento Livre e Esclarecido (TCLE) antes da participação, e os dados coletados de todos os sujeitos foram mantidos estritamente confidenciais.

Os dados foram coletados por meio do questionário (Quadro 1) baseado no trabalho de Santos e colaboradores (2016), e este foi desenvolvido para obter informações sobre o conhecimento teórico da ECG, como o objeto de avaliação e parâmetros avaliados (questões 1 e 2), e o conhecimento prático da ECG utilizando casos clínicos que demandam cálculo das variáveis (questões 3, 4 e 5).

A análise dos dados foi realizada pelo programa Microsoft Excel 2010, versão 14.0.72685000. 
Quadro 1. Questionário aplicado aos discentes.

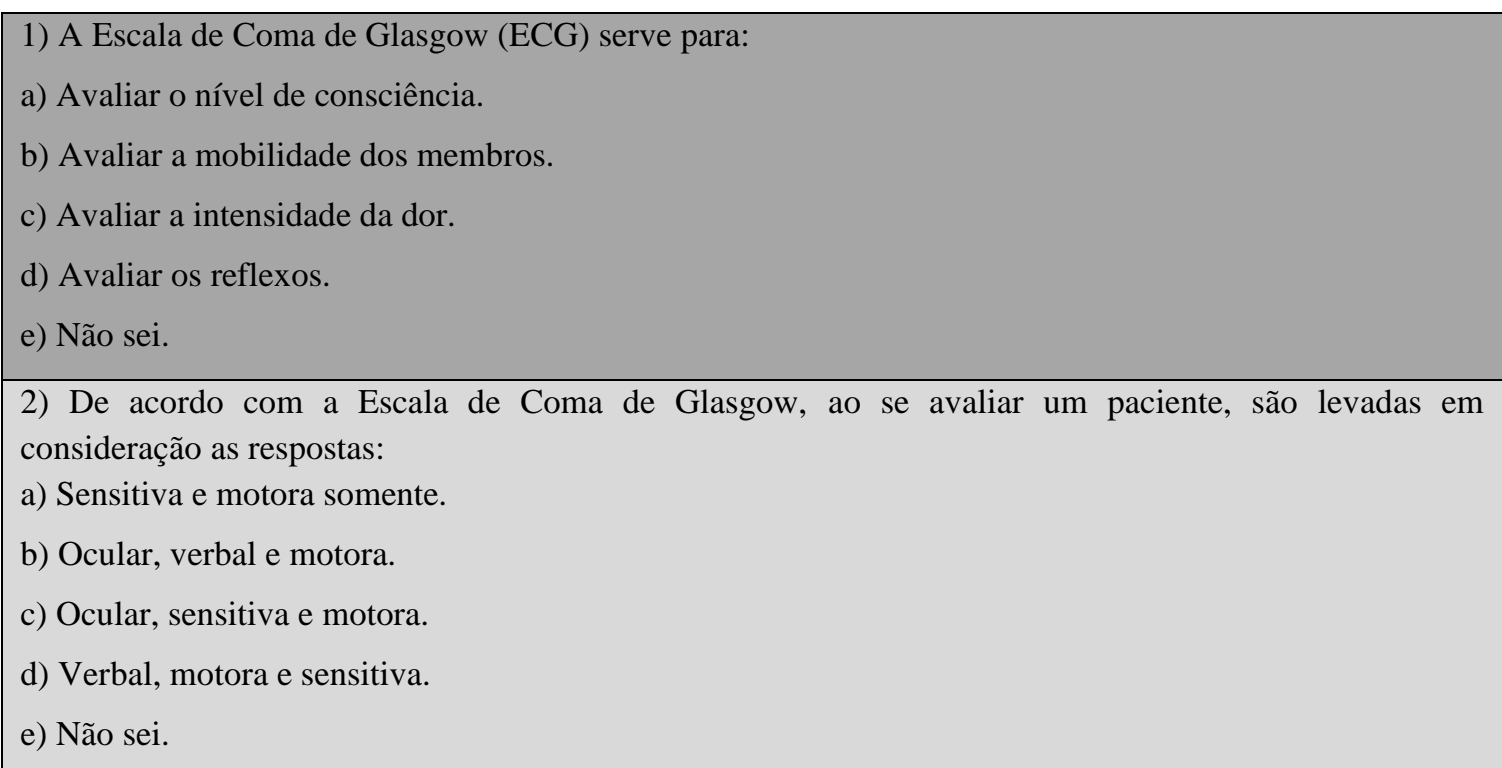

3) Um paciente de 30 anos, vítima de acidente automobilístico, foi internado na Unidade de Tratamento Intensivo. Ele apresenta abertura ocular ao estímulo à pressão, resposta verbal com sons ininteligíveis e flexão ao estímulo doloroso (decorticação). De acordo com os parâmetros da escala de coma de Glasgow, o nível de consciência desse paciente é compatível com:
a) 13 .
b) 12 .
c) 8 .
d) 7 .
e) Não sei.

4) Paciente politraumatizado deu entrada no serviço de emergência. Durante a análise do nível de consciência, foram observados os seguintes parâmetros: abertura ocular ao estímulo doloroso, resposta verbal com palavras inapropriadas e resposta motora com movimento de retirada. De acordo com a análise, o paciente encontra-se em qual nível na escala de coma de Glasgow?
a) 8 .
b) 9 .
c) 10 .
d) 11 .
e) Não sei.
a) Postura de decorticação. ECG 4.
b) Postura de decorticação. ECG 5.
c) Postura de descerebração. ECG 4.
d) Postura de descerebração. ECG 5.
e) Não sei.

5) O paciente vítima de traumatismo cranioencefálico que não abre os olhos, não emite sons, hiperestende os membros inferiores e superiores ao estímulo doloroso apresenta-se em: 


\section{Resultados e Discussão}

A aplicação do questionário aos acadêmicos dos anos finais dos cursos da área da saúde gerou os dados apresentados na

Tabela 2, tendo como número amostral 100 discentes.

Tabela 2. Distribuição dos participantes perante curso de graduação e gênero.

\begin{tabular}{c|c} 
Curso de Graduação & Número de Acadêmicos \\
N=100
\end{tabular}

Fonte: Autores.

Observou-se a predominância de alunos do curso de Farmácia (38\%) e de participantes do sexo feminino (67\%).

A Tabela 3 correlaciona às questões e a quantidade de acertos dos participantes em relação ao curso de graduação. 
Tabela 3. Número de participantes que acertaram as questões apresentadas por curso de graduação.

\begin{tabular}{|c|c|c|}
\hline Curso de Graduação & Questão & Número de acertos $(\%)$ \\
\hline \multirow{5}{*}{ Enfermagem } & 1 & $13(100 \%)$ \\
\hline & 2 & $12(92,31 \%)$ \\
\hline & 3 & $4(30,77 \%)$ \\
\hline & 4 & $9(69,23 \%)$ \\
\hline & 5 & $5(38,46 \%)$ \\
\hline \multirow{5}{*}{ Farmácia } & 1 & $28(73,68 \%)$ \\
\hline & 2 & $15(39,47 \%)$ \\
\hline & 3 & $6(15,79 \%)$ \\
\hline & 4 & $7(18,42 \%)$ \\
\hline & 5 & $7(18,42 \%)$ \\
\hline \multirow{5}{*}{ Medicina } & 1 & $20(100 \%)$ \\
\hline & 2 & $20(100 \%)$ \\
\hline & 3 & $18(90 \%)$ \\
\hline & 4 & $14(70 \%)$ \\
\hline & 5 & $18(90 \%)$ \\
\hline \multirow{5}{*}{ Odontologia } & 1 & $13(44,82 \%)$ \\
\hline & 2 & $2(6,9 \%)$ \\
\hline & 3 & $7(24,14 \%)$ \\
\hline & 4 & $7(24,14 \%)$ \\
\hline & 5 & $6(20,69 \%)$ \\
\hline
\end{tabular}

Fonte: Autores.

A Tabela 4 mostra o panorama geral da pesquisa e dos participantes, correlacionando a quantidade de acerto nas questões.

Tabela 4. Número de participantes/acertos por questão.

\begin{tabular}{cc} 
Questão & Número de Acertos (\%) \\
\hline 1- Função da ECG & $74(74 \%)$ \\
2- Parâmetros da ECG & $49(49 \%)$ \\
3- Cálculo das variáveis & $35(35 \%)$ \\
4- Cálculo das variáveis & $37(37 \%)$ \\
5- Cálculo das variáveis & $36(36 \%)$ \\
\hline
\end{tabular}

Fonte: Autores.

A maioria dos discentes (74\%) referiu corretamente à função da escala de coma de Glasgow, todos os graduandos entrevistados do curso de Medicina e Enfermagem pontuaram essa questão. Quando os participantes foram indagados sobre os 
parâmetros avaliados na ECG somente $49 \%$ marcaram a resposta ocular, verbal e motora, sendo estes em sua maioria do curso de medicina e enfermagem $(65,3 \%)$.

O cálculo das variáveis apresentadas nos casos clínicos das questões 3, 4 e 5 apresentaram os menores índices gerais de acerto, os discentes do curso de medicina mostraram melhor discernimento no cálculo dos parâmetros, obtendo acertos de $90 \%, 70 \%$ e $90 \%$, respectivamente. Seguidos pelos alunos de enfermagem com 30,77\% de acertos da questão 3, 69,23\% de acertos na questão 4 e 38,46\% na questão 5. Os alunos de farmácia e odontologia obtiveram os menores índices de acertos na aplicabilidade da ECG, variando de $15,79 \%$ a $24,14 \%$.

A avaliação do nível de consciência faz parte da rotina dos profissionais da área da saúde (Edwards, 2001; Ellis \& Cavanagh, 1992; Jain \& Iverson, 2021). O conhecimento e habilidade de uso da ECG são essenciais para estes profissionais, e a falha no uso da escala resulta em imprecisões na avaliação e conduta (Shoqirat, 2006), além de falhas na comunicação entre a equipe multiprofissional (Jain \& Iverson, 2021). A compreensão e habilidades de profissionais e alunos no uso da ECG dependem da educação recebida pelos praticantes (Elliott, 1996).

Este estudo sugere que os discentes formandos em Medicina apresentam um bom conhecimento teórico-prático da ECG, demonstrado pela quantidade de respostas corretas no questionário, tendo no mínimo $70 \%$ de acertos. Os estudos realizados por Emeleju (2014) e Riechers (2005), retratam que após a formatura os médicos tendem a esquecer da escala e sua utilização, principalmente aqueles que não trabalham diretamente com o meio cirúrgico ou atendimento de emergência, o que alerta para a importância da educação continuada e constante capacitação profissional.

Os discentes de Enfermagem obtiveram um bom desempenho teórico sobre a ECG, evidenciando seus conhecimentos sobre a função da escala (100\% de acertos) e parâmetros avaliados $(92,31 \%$ de acertos), entretanto demonstraram conhecimento moderado na aplicação da mesma, com índices de acerto de 30,77 a 69,23\% nas questões de cálculo das variáveis. Isso confirma as declarações de Shoqirat (2006), Igram (1994) e Hickey (1997), que os alunos e profissionais da enfermagem apresentam dificuldades no uso da ECG e a ferramenta não é vista como de responsabilidade da equipe de enfermagem.

Os discentes de Odontologia e Farmácia obtiveram um desempenho baixo na assertividade do questionário, apresentaram conhecimento da função da ECG- com 44,82\% e 73,68\%, respectivamente. Entretanto apresentaram baixa adesão as questões 2 a 5 , o que é evidenciado pela baixa porcentagem de acertos das mesmas, demonstrando assim desconhecimento sobre a existência e função da ECG e sua aplicabilidade.

Conforme Shoqirat (2006), frequentemente o acadêmico tem a visão de que o conhecimento e aplicação da ECG é dever do médico. Com a importância dada a interdisciplinariedade do atendimento hospitalar (Fernades, 2019), a participação ativa de médicos e corpo de enfermagem, juntamente com fisioterapeutas (Guimarães, 2020), dentistas (Aranega et al., 2012) e farmacêuticos (Fernandes, 2019) são essenciais para o cuidado, sendo assim todos os profissionais devem conhecer a ECG e saber aplica-la, para fornecer ao paciente um monitoramento seriado e evitar problemas de comunicação entre a equipe (Jain \& Iverson, 2021).

A falta de conhecimento adequado da aplicação desta ferramenta impacta negativamente no atendimento de pacientes e no serviço de saúde (Emeleju, 2014). Sendo de suma importância que durante a graduação o acadêmico tenha contato com a escala por meio de uma educação teórica e treinamento prático eficaz (Shoqirat, 2006), e após a formação o treinamento continuado dos profissionais é crucial na excelência do cuidado em saúde (Reith et al., 2016). 


\section{Conclusão}

A maioria dos discentes do curso de medicina e enfermagem apresenta bom conhecimento teórico da escala de coma de Glasgow, entretanto somente os discentes de medicina demonstram um bom conhecimento da aplicabilidade e dos cálculos da ECG. Os acadêmicos dos demais cursos pesquisados apresentam déficits de conhecimentos sobre o tema.

O déficit de conhecimento sobre a escala de coma de Glasgow deve ser suprido dentro das universidades, por meio de treinamentos teóricos e práticos. Haja vista que o conhecimento pleno da ECG e sua aplicação por toda a equipe multiprofissional são essenciais na efetividade e excelência do serviço de saúde.

Confirmando a impressão da literatura sobre discrepância no conhecimento e aplicação da ECG entre os diversos profissionais da saúde, outros estudos são necessários para verificar a eficácia do contínuo aprendizado teórico e prático sobre o uso e aplicação da escala de coma de Glasgow.

\section{Agradecimentos}

Os autores agradecem a Universidade Federal de Alfenas por sediar a pesquisa e aos estudantes formandos dos cursos de Medicina, Farmácia, Enfermagem e Odontologia que forneceram os dados para o estudo.

\section{Referências}

ATLS, Advanced Trauma Life Suport (2018). American college of surgions committee on trauma. Livro básico.

Aranega, A. M., Bassi, A. P. F., Ponzoni, D., Wayama, M. T., Esteves, J. C., \& Garcia Jr., I. R. (2012). Qual a importância da Odontologia Hospitalar? Revista Brasileira de Odontologia, 69(1), 90-93.

Baker, M. (2008). Reviewing the application of the Glasgow Coma Scale: Does it have interrater reliability? British Journal of Neuroscience Nursing. 4 (7) $342-347$.

Brennan, P. M., Murray, G. D., \& Teasdale, G. M. (2018). Simplifynd the use of prognostic information in traumatic brain injury. Part 1: The GCS-Pupils score: an extended index of clinical severity. Journal of Neurosurgery, 128, 1612-1620.

Bryan, J. (2002). The Glasgow coma scale: History and current practice. Trauma, 4 (1) 91-103.

Edwards, S. L. (2001). Using the Glasgow Coma Scale: analysis and limitations. British Journal of Nursing, 10 (2), $92-101$.

Elliott, M. (1996). Interrater reliability of the Glasgow Coma Scale. Journal of Neuroscience Nursing, 28(4), $213-214$.

Ellis, A, \& Cavanagh, S. J. (1992). Aspects of neurosurgical assessment using the Glasgow Coma Scale. Intensive and Critical Care Nursing, 8 (2), 94-99.

Emeleju, J. K. C., Nkwerem, S. P. U., \& Ekweogwu, O. C. (2014). Assessment of physicians' knowledge of Glasgow coma score. Nigerian Journal of Clinical Practice. 17 (6), 729-734.

Fernandes, L. L. (2019). A importância do farmacêutico hospitalar juntamente com a equipe multidisciplinar na Unidade de Terapia Intensiva (UTI). Revista Farol. 8 (8), 5-21.

Fischer, J., \& Mathieson, C. (2001). The history of the Glasgow coma scale: Implications for practice. Critical Care Nursing Quarterly, 23 (1), 52-58.

Guimarães, F. (2020). Atuação do fisioterapeuta em unidades de terapia intensiva no contexto da pandemia de COVID-19. Fisioterapia em Movimento, 33, 13 .

Hickey, J. V. (1997). The Clinical Practice of Neurological and Neurosurgical Nursing. Livro básico.

Ingram, N. (1994). Knowledge and level of consciousness: Application to nursing practice. Journal of Advanced Nursing, 20 (5), $881-884$.

Jain, S, \& Iverson, L. (2021). Glasgow Coma Scale. Treasure Island (FL): StatPearls Publishing. https://www.ncbi.nlm.nih.gov/books/NBK513298/

Jennett, B, \& Teasdale, G (1997). Aspects of coma after severe head injury. Lancet, 1 (8017), 878-881.

Mattar, I., Liaw, S. Y., \& Chan, M. F. (2013). A study to explore nurses' knowledge in using the Glasgow Coma Sclae in na Acute Care Hospital. Journal of Neuroscience Nursing. 45 (5), 272-280.

Mcnarry, A. F., \& Goldhill, D. R. (2004). Simple bedside assessment of level of consciousness: comparison of two simple assessment scales with the Glasgow Coma Scale. Anaesthesia, 59 (1), 34-37. 
Research, Society and Development, v. 10, n. 9, e2410917798, 2021

(CC BY 4.0) | ISSN 2525-3409 | DOI: http://dx.doi.org/10.33448/rsd-v10i9.17798

Reith, F. C., Brennan, P M., Maas, A. I, \& Teasdale, G. M. (2016). Lack of Standardization in the Use of the Glasgow Coma Scale: results of International Surveys. Journal of Neurotrauma, 33 (1), 89-94.

Riechers, R. G., et al. (2005). Physician Knowledge of the Glasgow Coma Scale. Journal of Neurotrauma, 22 (1), 1327-1324.

Ross, S. E., Leipold, C. R. N., Terregino, C., \& O'malley, K. F. (1998). Efficacy of the Motor Component of the Glasgow Coma Scale in Trauma Triage. The Journal of Trauma. 45 (1), $42-44$.

Santos, W. C., Vancini-Campanharo, C. R., Lopes, M. C. B. T., Okuno, M. F. P., \& Batista, R. E. A. (2016). Avaliação do conhecimento de enfermeiros sobre a escala de coma de Glasgow em um hospital universitário. Einstein, 14 (2), 213-218.

Shah, S. (1999). Neurological assessment. Nursing Standard, 13 (22), 49-54.

Shoqirat, N. (2006). Nursing students’ understanding of the Glasgow Coma Scale. Art \& Science, 20 (30), 41-47.

Stewart, N. (1996). Neurological Observations. Professional Nurse, 11 (6), 377-378. 